FECHA DE RECEPCIÓN: 13/01/2019 FECHA DE APROBACIÓN: 24/04/2019 FECHA PUBLICACIÓN: 10/09/2019

1. Hospital Vicente Corral Moscoso

2. Universidad de Cuenca

\begin{tabular}{r|l} 
Caso & Clinical \\
Clínico & Case
\end{tabular}

DOI: https://doi.org/10.18537/RFCM.37.02.06 Correspondencia: dforellanac891ahotmail.com

Dirección:

Juan Jaramillo 4-10

Código Postal:

010104

Celular:

0984276134

Cuenca - Ecuador

\section{Insuficiencia cardiaca secundaria a hipocalcemia asociada a hipoparatiroidismo: reporte de un caso}

\author{
Heart failure secondary to hypocalcemia associated with \\ hypoparathyroidism: a case report \\ Orellana Cobos Danilo Fernando', Tigsi Mendoza Carlos Alfredo', \\ León Astudillo Edgar José1, Álvarez Palacios Franklin Santiago', \\ Álvarez Serrano Marlene Elizabeth², Rivas Ullaguari Ana Belén', \\ Sacoto Molina Adrián Marcelo²
}

\section{RESUMEN}

La insuficiencia cardiaca debida a hipocalcemia es una extraña forma de cardiopatía metabólica, reversible en la mayoría de casos, secundaria a alteraciones endocrinológicas que provocan la disminución persistente del calcio sérico, como el hipoparatiroidismo. A continuación, se presenta el caso de una mujer de 23 años, a quien, al ingreso, se le diagnóstico de insuficiencia cardiaca severa. Durante su hospitalización, se evidenció sintomatología asociada con hipocalcemia, secundario a hipoparatiroidismo, por lo que inmediatamente se inició la reposición con calcio. Para prevenir el riesgo de muerte súbita, por la prolongación persistente del intervalo Q-T, se colocó un cardiodesfibrilador implantable; sin embargo, luego de 6 meses de tratamiento, a pesar de la suplementación con calcio oral, no se evidenció mejoría en la fracción de eyección, determinando un pronóstico incierto de la enfermedad

Palabras clave: insuficiencia cardíaca, hipoparatiroidismo; hipocalcemia.

\section{ABSTRACT}

Heart failure due to hypocalcemia is a strange presentation of metabolic heart disease; it is reversible in most cases, and secondary to endocrinal alterations that cause the persistent decrease in serum calcium, such as hypoparathyroidism.

A case of a 23-year-old woman who was diagnosed with severe heart failure is presented. During her hospitalization, there was evidence of symptoms associated with hypocalcemia, secondary to hypoparathyroidism, so the patient was treated with replacement of calcium immediately. To prevent the risk of sudden death, by the persistent prolongation of the Q-T interval, an implantable cardiac defibrillator was placed; however, after 6 months of treatment, despite oral calcium supplementation, there was no improvement in the ejection fraction, determining an uncertain prognosis of the disease.

Keywords: heart failure, hypoparathyroidism, hypocalcemia. 


\section{INTRODUCCIÓN}

El hipoparatiroidismo primario (HPTP) se caracteriza por una producción insuficiente de hormona paratiroidea, secundaria en la mayoría de ocasiones a su extirpación en las cirugías de cuello (tiroidectomías), o en menor cantidad, a causas genéticas o autoinmunes [1].

La paratohormona (PTH) regula el metabolismo del calcio y fósforo a través de varias rutas, por lo que su deficiencia provoca un amplio espectro de manifestaciones clínicas dependientes de la hipocalcemia resultante, relacionadas principalmente con la contractilidad muscular. A nivel cardiaco, el calcio es el principal determinante de la contractilidad miocárdica, por lo que su disminución en sangre puede conducir a prolongación del intervalo $Q-T$, hipotensión, arritmias, y en casos raros, insuficiencia cardiaca [2].

El diagnóstico se basa en la presencia de hipocalcemia persistente, asociada hiperfosfatemia en ausencia de alteraciones del magnesio sérico, secundario a un nivel bajo de PTH $[3,4]$.

A continuación, exponemos el caso de una paciente con insuficiencia cardiaca severa debido a hipocalcemia secundaria a hipoparatiroidismo; poco frecuente en la práctica clínica. Al momento son pocos los casos documentados de esta patología, siendo importante el reporte del presente caso para incluir a las alteraciones metabólicas, como parte del diagnóstico etiológico diferencial de la insuficiencia cardíaca.

\section{PRESENTACIÓN DEL CASO}

Paciente femenina, de 23 años, soltera, procedente de Machala - Ecuador, residente en Cuenca - Ecuador, sin ocupación actualmente, acudió al servicio de emergencia por presentar edema de miembros inferiores.

Refirió que, desde hace 3 semanas, previo a su ingreso, presentó edema de miembros inferiores que fue aumentando progresivamente, sumándose disnea de grado II que progresó grado III. Una hora previa a su ingreso, presentó síncope, por lo cual fue traída al servicio de emergencias del Hospital Regional Vicente Corral Moscoso.
Dentro de los antecedentes patológicos personales refirió presentar desde hace 6 meses, crisis convulsivas tónico clónicas generalizadas, intermitentes, sin revisión médica ni tratamiento instaurado. Además, indicó dentro de los antecedentes familiares, que la madre falleció por una patología cardiaca no especificada.

Al examen físico, presentaba una frecuencia respiratoria de 26 por minuto, con una saturación de oxígeno del $96 \%$ con FiO2 28. Talla de $169 \mathrm{~cm}$, sin alteración auditiva, olfatoria ni visual. Ingurgitación yugular con un soplo sistólico en foco tricuspídeo y diastólico en foco aórtico. Hepatomegalia de 2 centímetros por debajo de borde costal y edema frío en miembros inferiores con fóvea de tres cruces, que ascendía hasta el muslo.

Al ingreso la paciente presentó la siguiente analítica (Tabla $\mathrm{N}^{\circ} 1$ ), evidenciándose una hiponatremia leve, con prolongación de los tiempos de coagulación, hipoalbuminemia y elevación de enzimas hepáticas. 


\section{TABLA Nº 1}

Exámenes de laboratorio al ingreso de la paciente

\begin{tabular}{|c|c|c|c|c|c|}
\hline Parámetro & Valor & Referencia & Parámetro & Valor & Referencia \\
\hline \multicolumn{3}{|c|}{ Biometría hemática } & \multicolumn{3}{|c|}{ Química sanguínea } \\
\hline G. Blancos & 7.2 & $5.0-10.0$ & TP & 30.1 & $10.0-13.5$ \\
\hline Neutrófilos & $58.3 \%$ & $50-67$ & INR & 2.7 & $0.0-2.0$ \\
\hline Linfocitos & $34 \%$ & $28.0-44.0$ & TPT & 38.4 & $20-45$ \\
\hline Hemoglobina & 12.1 & $12.5-14.7$ & Glucosa & 128.0 & $70-100$ \\
\hline Hematocrito & 35.4 & $40.0-47.0$ & $\begin{array}{l}\text { Proteínas } \\
\text { totales }\end{array}$ & 6.7 & $6.6-8.7$ \\
\hline G. Rojos & 3.9 & $4.6-5.2$ & Albumina & 3.4 & $3.5-5.2$ \\
\hline Plaquetas & 140 & $140-450$ & Globulina & 3.4 & $2.0-3.0$ \\
\hline \multicolumn{3}{|c|}{ Electrolitos } & TGO & 638.0 & $0.0-32.0$ \\
\hline $\mathrm{Na}$ & 131 & $135-148$ & TGP & 446.8 & $0.0-35.0$ \\
\hline K & 4.33 & $3.5-5.3$ & GGT & 153.0 & $10.0-47.0$ \\
\hline \multirow[t]{2}{*}{$\mathrm{Cl}$} & 91.5 & $98-110$ & LDH & $2,745.0$ & $120-250$ \\
\hline & & & FALC & 152.0 & $40-129$ \\
\hline
\end{tabular}

Elaborado por: los autores

Fuente: historia clínica

Se realizó una radiografía estándar de tórax (Imagen $N^{\circ} 1$ ), donde se evidenció una cardiomegalia grado IV, con un índice cardio - torácico de 0.7 , a confirmarse presumiblemente una insuficiencia cardiaca.

\section{Imagen $\mathrm{N}^{0} 1$}

Radiografía estándar de tórax

Se observa cardiomegalia grado IV con un índice cardio - torácico de 0.7 , además de congestión hiliar y reforzamiento de la trama vascular

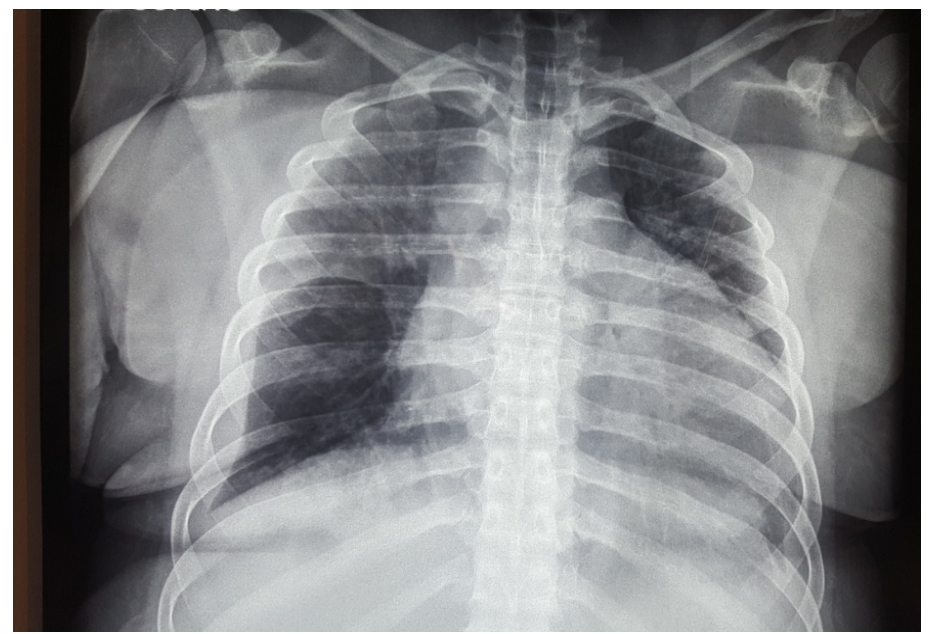

La ecografía abdominal reportó un hígado congestivo con dilatación de las venas suprahepáticas, además de derrame pleural derecho leve. En el electrocardiograma se evidenció un ritmo sinusal, con frecuencia cardiaca de 96 latidos por minuto, un eje cardiaco desviado a la derecha y un intervalo $\mathrm{Q}-\mathrm{T}$ corregido para la frecuencia cardiaca, de 556 milisegundos (Imagen $N^{\circ} 2$ ). 


\section{Imagen $\mathrm{N}^{\circ} 2$}

\section{Electrocardiograma de 12 derivaciones}

Se evidencia ritmo sinusal, con frecuencia cardiaca de 96 latidos por minuto. Las flechas indican el intervalo Q-T prolongado (de 556 milisegundos) observado en todas las derivaciones

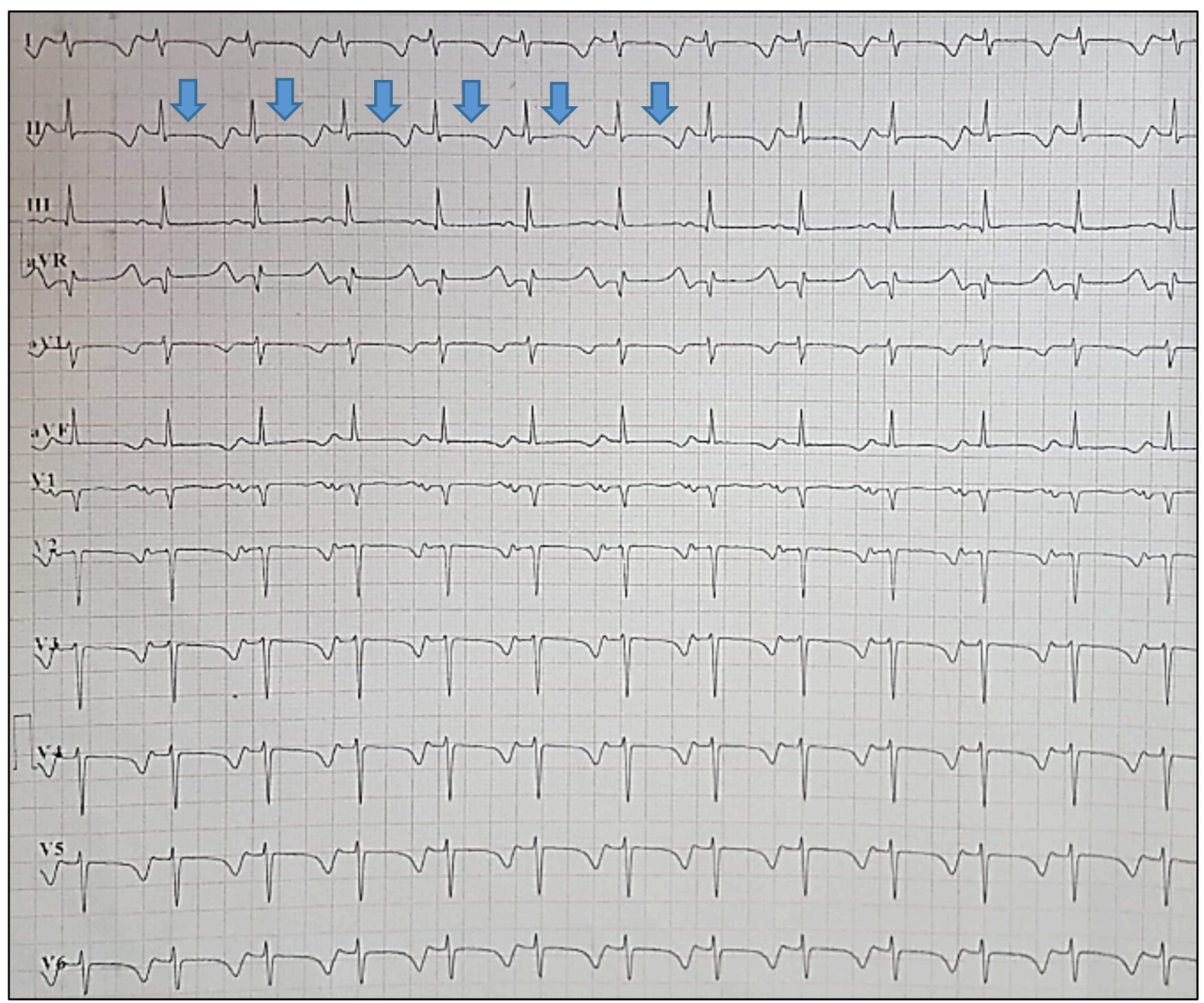

Se realizó una ecocardiografía que reportó dilatación del ventrículo izquierdo con disfunción sistólica severa, con una fracción de eyección del 13\%; dilatación de la aurícula izquierda, y ventrículo derecho aumentado de tamaño, con signos de disfunción sistólica, TAPSE $1.0 \mathrm{~cm}$ con regurgitación tricuspídea y un gradiente máximo de $30 \mathrm{mmHg}$.

Al tercer día de hospitalización, la paciente presentó una crisis convulsiva tónico clónica generalizada, por lo que se solicitó, entre los múltiples exámenes de laboratorio, la dosificación de calcio plasmático, reportándose un valor de 2.8 miligramos/decilitro (calcio corregido para la albumina de 3.52 miligramos/decilitro). Al examen físico destacaba la presencia de los signos de Trousseau y Chvostek. Se realizó una tomografía simple de cráneo (Imagen $N^{\circ} 3$ ), encontrándose calcificación bilateral de ganglios basales y plexos coroideos, con depósitos visibles de calcio en cerebelo y múltiples calcificaciones diseminadas en masa encefálica; lo cual orientaba a una alteración del metabolismo cálcico. 


\section{Imagen $\mathrm{N}^{\circ} 3$}

\section{Tomografía axial computada de cráneo}

En las imágenes $A$ y $D$ se observa una difusa calcificación de los ganglios basales, de manera simétrica. En la imagen B se aprecia calcificación marcada de los plexos coroideos. En las imágenes C y E se distinguen depósitos de calcio a nivel de la corteza cerebelosa
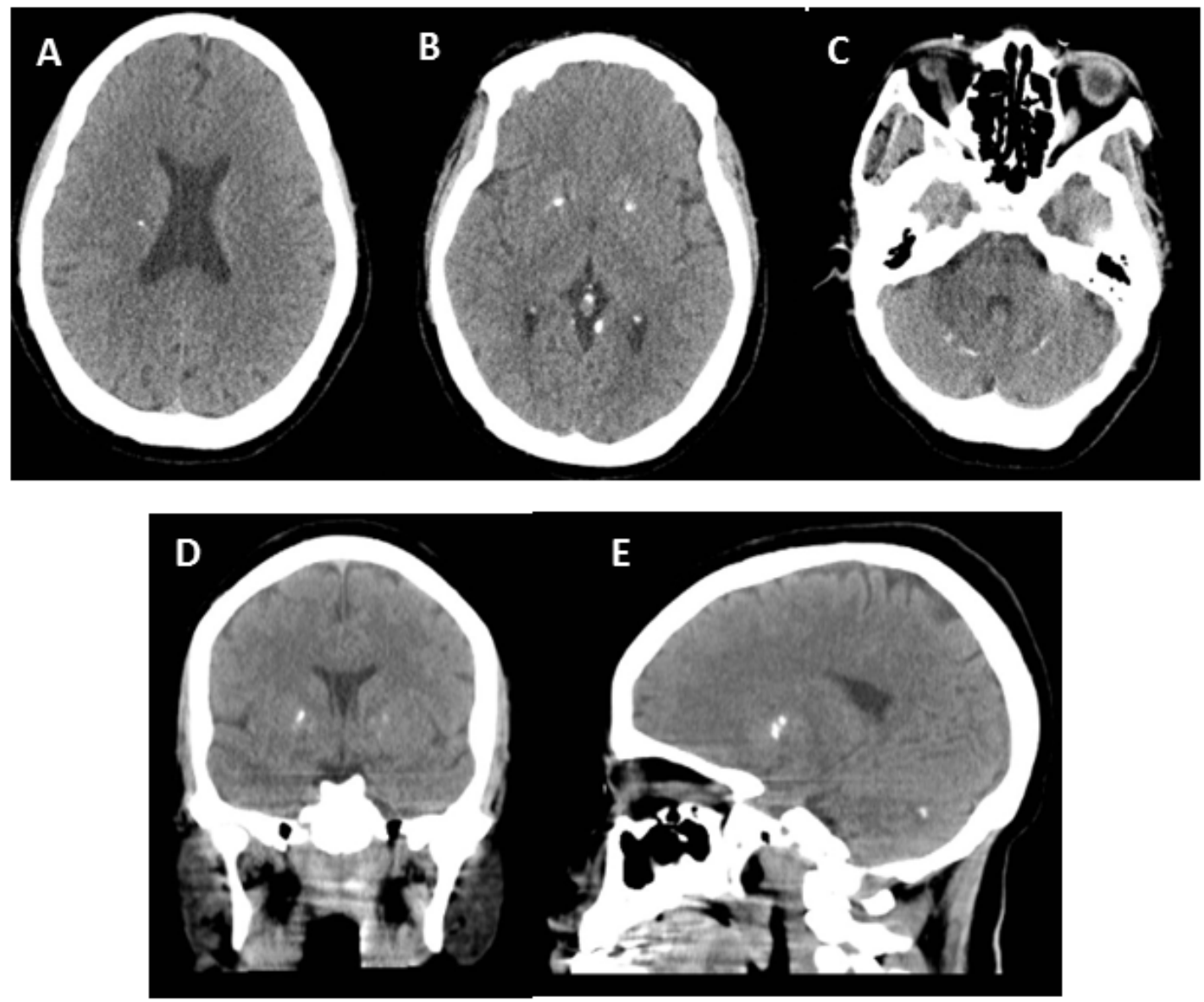

\section{EVOLUCIÓN Y TRATAMIENTO}

En base a la analítica y resultados obtenidos, se instauró la terapéutica específica basada en dos pilares: el tratamiento de la insuficiencia cardiaca (con betabloqueante, IECA, diurético de asa); y la reposición y mantenimiento de los niveles de calcio sérico. Se interconsultó con el servicio de endocrinología quienes solicitaron la dosificación en suero de paratohormona, la cual reportó un valor de 5.6 picogramos/mililitro. Durante su estancia hospitalaria se mantuvo en seguimiento por la especialidad, con el fin de mantener los niveles de calcio sérico dentro de rangos normales, con la suplementación de carbonato de calcio y calcitriol. De igual manera se interconsultó con el departamento de genética de la unidad, quienes luego de la valoración respectiva sugirieron, en base a los signos clínicos y radiológicos, la posibilidad de una calcinosis bilateral de los ganglios basales con un hipoparatiroidismo asociado e insuficiencia cardiaca severa. Fue valorada por el servicio de cardiología quienes, para disminuir el riesgo de muerte súbita y las complicaciones por la fracción de eyección reducida, propusieron la colocación de un cardiodesfibrilador implantable, el cual se llevó a cabo exitosamente, sin complicaciones.

Posterior al procedimiento, la paciente fue dada de alta, en mejores condiciones, con oxígeno suplementario y calcio sérico normal (calcio corregido $=$ 9.1 miligramos/decilitro). Luego de 6 meses de tratamiento a base de suplementación con calcio oral, se realizó un nuevo ecocardiograma donde se evidenció la persistencia de la disfunción sistólica del ventrículo izquierdo, manteniéndose una fracción 
de eyección disminuida; por lo que, al momento, el pronóstico es incierto.

\section{DISCUSIÓN}

La presentación clínica del hipoparatiroidismo es diversa, dependiendo las manifestaciones clínicas de la hipocalcemia secundaria, provocando alteraciones del sistema neuromuscular principalmente [5]. De manera aguda, puede presentarse parestesias de miembros, hiperreflexia e incluso tetania o laringoespasmo en los casos graves [6]. A nivel cardíaco, es muy frecuente encontrar prolongación del intervalo Q-T, siendo raro la aparición de insuficiencia cardiaca; la misma, que en caso de presentarse, suele ser refractario al tratamiento convencional [7]. En el caso de la paciente, la primera manifestación de la hipocalcemia secundaria a su hipoparatiroidismo fue la insuficiencia cardiaca, constituyendo un debut inusual de la enfermedad, ya con repercusión hepática, evidenciado en la analítica y la ecografía abdominal. Realizando una anamnesis dirigida, se encontró el antecedente de crisis convulsivas; las mismas que presumiblemente se debieron a estados de hipocalcemia previos, o incluso a las calcificaciones cerebrales presentes en la tomografía; lo cual concuerda con la calcinosis intracerebral presente en los estados de hipoparatiroidismo crónico sin tratamiento. De igual manera, los signos de Chvostek y Trousseau presentes luego de una crisis convulsiva presenciada durante su hospitalización, orientaron a la presunción diagnóstica

La principal causa de hipoparatiroidismo es la iatrogénica, debida a la resección quirúrgica en las tiroidectomías [8]. Las causas autoinmunitarias ocupan el segundo lugar en frecuencia, pudiendo presentarse asociada a un síndrome poliglandular autoinmune tipo 1; o de manera aislada, debido a la presencia de anticuerpos anti-CaSr (poco estudiado hasta el momento) [9]. Aún más extraño, constituyen las causas genéticas o el pseudohipoparatiroidismo, donde una resistencia a la acción de la PTH provoca hipocalcemia e hiperfosfatemia con valores anormalmente elevados de paratohormona, en ausencia de enfermedad renal [10]. En el caso de la paciente, la causa iatrogénica se descarta por la ausencia de un antecedente quirúrgico previo; al igual que causas que provoquen la destrucción de la glándula, como la radiación, infiltración metastásica o la exposición a metales. La ausencia de candidiasis mucocutánea crónica o insuficiencia suprarrenal asociada, descarta la presencia de un síndrome poliglandular autoinmune; dejando abierta la opción de un hipoparatiroidismo aislado debido a una activación de anticuerpos contra el receptor de los sensores de calcio (anti-CaSr), cuya característica principal es su debut en la edad adulta, cuando de manera incidental se determinan valores de calcio sérico bajos [11].

En relación al tratamiento, se ha evidenciado que la reposición de los niveles de calcio sérico dentro de valores normales, revierte la cardiopatía hipocalcémica; mejorando la función cardiaca con un incremento progresivo de la fracción de eyección $[12,13]$. Mientras más temprano se realice el diagnóstico y se implementa la terapéutica adecuada, se ha observado mejores resultados en la reversibilidad del cuadro [14]. En el caso de nuestra paciente, la prolongación del intervalo Q-T aumentaba el riesgo de muerte súbita; por lo que para prevenir aquello, se decidió la colocación de un cardiodesfibrilador implantable, con lo cual se consiguió mejoría en el electrocardiograma; no obstante, no se evidenció una recuperación adecuada de la fracción de eyección luego de 6 meses de tratamiento con la reposición de calcio, lo cual constituye un factor de mal pronóstico [15].

\section{CONCLUSIONES}

La insuficiencia cardíaca secundaria a hipocalcemia constituye una rara causa de cardiopatía metabólica reversible, asociada generalmente a enfermedades endocrinológicas, como el hipoparatiroidismo. El restablecimiento de la funcionalidad cardíaca se ha logrado con un diagnóstico y tratamiento oportuno en la mayoría de casos reportados por la literatura; no obstante, en el caso presentado, no se evidencia una evolución favorable del cuadro, lo cual empobrece el pronóstico de la paciente. De ahí, la importancia de incluir en el diagnóstico diferencial de la insuficiencia cardíaca a las enfermedades endocrino - metabólicas, cuyo diagnóstico precoz permitirá mejorar la evolución natural de la enfermedad [16].

\section{ASPECTOS BIOÉTICOS}

El presente trabajo se realizó bajo el consentimiento informado de la paciente, con total confidencialidad de datos personales. 


\section{INFORMACIÓN DE LOS AUTORES}

- Orellana Cobos Danilo Fernando. Médico General. Hospital Vicente Corral Moscoso. Clínica. Medicina Interna. Cuenca - Azuay - Ecuador e-mail: dforellanac891@hotmail.com ORCID: http://orcid.org/0000-0001-6269-5512

- Tigsi Mendoza Carlos Alfredo. Especialista en Endocrinología. Hospital Vicente Corral Moscoso. Clínica. Endocrinología. Cuenca - Azuay Ecuador.

e-mail: carlitostigsi@gmail.com ORCID: http://orcid.org/0000-0002-0181-786X

- León Astudillo Edgar José. Especialista en Endocrinología. Hospital Vicente Corral Moscoso. Clínica. Endocrinología. Cuenca - Azuay Ecuador.

e-mail: edgarjose26@hotmail.com

ORCID: https://orcid.org/0000-0002-2268-2255

- Álvarez Palacios Franklin Santiago. Especialista en Medicina Interna. Hospital Vicente Corral Moscoso. Clínica. Medicina Interna. Cuenca Azuay - Ecuador.

e-mail: santiago300884@hotmail.com

ORCID: https://orcid.org/0000-0003-3104-5407

- Álvarez Serrano Marlene Elizabeth. Especialista en Medicina Interna. Universidad de Cuenca. Facultad de Ciencias Médicas. Carrera de Medicina. Cuenca - Azuay - Ecuador.

e-mail: marlene.alvarezs@ucuenca.edu.ec

ORCID: http://orcid.org/0000-0002-04726-8050

- Rivas Ullaguari Ana Belén. Médico General. Hospital Vicente Corral Moscoso. Clínica. Medicina Interna. Cuenca - Azuay - Ecuador.

e-mail: rivas.anabelen94@gmail.com

ORCID: http://orcid.org/0000-0002-6460-6250

- Sacoto Molina Adrián Marcelo. Magister en Investigación de la Salud. Universidad de Cuenca. Facultad de Ciencias Médicas. Carrera de Medicina. Cuenca - Azuay - Ecuador.

e-mail: asacoto7@gmail.com

ORCID: http://orcid.org/0000-0002-4048-9942

\section{CONTRIBUCIÓN DE LOS AUTORES}

Los autores declaran haber contribuido de manera similar en la concepción, diseño, análisis e interpretación de los datos, redacción del manuscrito y aprobación de la versión final, así como estar en capacidad de responder de todos los aspectos del mismo.

\section{CONFLICTO DE INTERESES}

Los autores no reportan conflicto de intereses.

\section{FUENTES DE FINANCIAMIENTO}

Autofinanciado.

\section{REFERENCIAS BIBLIOGRÁFICAS}

1. Velayuthan S, Gungor N, McVie R. Hypocalcemic cardiomyopathy as initial presentation of primary hypoparathyroidism. Pediatr Int. 2014;56(4):e23-5.

2. Kasper D, Hauser S, Jameson J, Fauci A, Longo D, Loscalzo J. Harrison. Principios de Medicina Interna. Trastornos de las glándulas paratiroides y la homeostasis de calcio. 19. ${ }^{a}$ ed. Vol. 3. USA: McGraw-Hill Medical; 2015. 2466-2487 p.

3. Román-González A, Zea-Lopera J, LondoñoTabares S, Builes-Barrera C, Sanabria A. Pilares para el enfoque y tratamiento adecuadodel pacienteconhipoparatiroidismo. IATREIA. 2018;31(2):155-65.

4. Sinnott BP. Hypoparathyroidism - Review of the Literature 2018. J Rare Disord Diagn Ther. 2018;04(03).

5. Clarke $B, M$ Brown $E$, Collins $M$, Jüppner $\mathrm{H}$, Lakatos $\mathrm{P}$, Levine $\mathrm{M}$, et al. Epidemiology and Diagnosis of Hypoparathyroidism. J Clin Endocrinol Metab. 2016;101.

6. Pacheco J, Guerrero $\mathrm{Y}$, Pedrique G, Pérez J, Zerpa Y. Manejo de pacientes con hipoparatiroidismo: Guías clínicas del Servicio de Endocrinología del Instituto Autónomo Hospital Universitario de Los Andes. Rev Venez Endocrinol Metab. 2014;12(3).

7. Kadeli D, Keshava R, Aniyathodiyil G, Reddy S, Magesh B, Prasanth K. Cardiomyopathy Secondary to Hypocalcemia. J Assoc Physicians India. 2018;66:2.

8. Wheeler A, Shoback D. Clinical Presentation of Hypoparathyroidism. En: Hypoparathyroidism. 1. ${ }^{\text {a }}$ ed. USA: M.L. Brandi. E.M. Brown; 2015. p. 155-65. 
9. Maeda SS, Moreira CA, Borba VZC, Bandeira F, Farias MLF de, Borges JLC, et al. Diagnosis and treatment of hypoparathyroidism: a position statement from the Brazilian Society of Endocrinology and Metabolism. Arch Endocrinol Metab. 2018;62(1):106-24.

10. Huang SH, Briceño $\mathrm{Y}$, Barrios MC, Silvestre $\mathrm{R}$, Paoli M. Resistencia a la paratohormona como causa infrecuente de hipocalcemia de inicio tardío en pacientes pediátricos: reporte de un caso. Rev Venez Endocrinol Metab. 2015;13:6.

11. Goltzman D, Cole D. Hypoparathyroidism. Primer on the Metabolic Bone Diseases and Disorders of Mineral Metabolism. Philadelphia, Pa: Lippincott-Raven; 1996: p. 220-22

12. Brown SJ, Ruppe MD, Tabatabai LS. The Parathyroid Gland and Heart Disease. Methodist DeBakey Cardiovasc J. 2017;13(2):49-54.

13. Bollerslev J, Rejnmark L, Marcocci C, Shoback DM, Sitges-Serra A, van Biesen W, et al. European Society of Endocrinology Clinical Guideline: Treatment of chronic hypoparathyroidism in adults. Eur J Endocrinol. 2015;173(2):G1-20.

14. Inomata T. Parathyroid Hormone in Heart Failure. Circ J. 2014;78(11):2631-2.

15. Çakërri L, Husi G, Minxuri D, Roko E, Vyshka G. Primary hypoparathyroidism presenting with heart failure and ventricular fibrillation. Oxf Med Case Rep. 2014;2014(4):77-9.

16. Burguez S. Insuficiencia cardíaca aguda. Rev Urug Cardiol. 2017;32(3). 